\title{
Optical properties of gallium phosphide (GaP) nanowires
}

\author{
Satyendra Singh $\cdot$ Pankaj Srivastava
}

Received: 1 November 2011/Accepted: 19 March 2012/Published online: 3 April 2012

(C) The Author(s) 2012. This article is published with open access at Springerlink.com

\begin{abstract}
The linear and non-linear optical properties of different geometrical structures of gallium phosphide $(\mathrm{GaP})$ nanowires have been studied by employing ab initio method. We have calculated the optical response of four different GaP nanowires, viz., two atom linear wire, two atom zigzag wire, four atom square wire and six atom hexagonal wire. We have investigated imaginary part of the $z z$ component of the linear dielectric tensor and second order susceptibility for different structures along with bulk material. We revealed that strongest absorption occurs for four atom square nanowire configuration.
\end{abstract}

Keywords Gallium phosphide nanowires · Linear and non-linear optical properties

\section{Introduction}

Optical properties of nanowires have become a major area of research for optoelectronic devices. When an electron absorbs a photon from the incident light, it makes a transition to the next higher unoccupied state and emits a photon of frequency less than or equal to the frequency of incident light; this phenomenon is known as linear optical transitions. It is found that there is significant shift in the optical absorption spectra toward the shorter wavelengths referred to as the blue shift as the particle size is reduced.

\section{S. Singh $(\bowtie)$}

Department of Physics, Hindustan College of Science

and Technology, Farah, Mathura 281122, India

e-mail: satyendra7171@yahoo.co.in

\section{P. Srivastava}

Applied Physics Group, Indian Institute of Information

Technology (ABV-IIITM), Gwalior 474010, India
The existence of the exciton has a strong influence on the electronic properties of the semiconductor and its optical absorption. The second-order response or non-linear property is a two photon process where the excited electron absorbs another photon of the same frequency and makes a transition to another allowed state at higher energy. When this electron is falling back to its original state, it emits a photon of frequency, which is twice the frequency of that of the incident light (Srivastava and Singh 2008). A linear phenomenon is somewhat simple but non-linear phenomenon corresponds to the appearance of a frequency component in the intense light beam that is exactly twice the input. The high aspect ratio of 1-D semiconductors gives rise to anisotropy in the electronic and optical properties; it may further enhance the long range dipolar interaction and produces significant changes in the transition states. Semiconducting nanowires have received much attention due to their potential application as building blocks of miniaturized electrical (Wang et al. 2005), nanofluidic (Karnik et al. 2005) and optical devices (Sirbuly et al. 2005). Nanowire photonic circuitry made from 1-D nanostructures offers numerous opportunities for the development of next generation optical information processors. The wave guiding property of individual nanowires depends on the wavelength of the emitted light.

Gallium phosphide $(\mathrm{GaP})$ is a semiconducting compound with an indirect band gap of $2.26 \mathrm{eV}$. It is one of the most promising optical materials having refractive index of about 3.37 in the visible region and it decreases to 3.2 in the infra red region. Thus, it can be utilized for manufacturing low and standard brightness, i.e., red, orange and green light emitting diodes (LED). It is also a low cost material with excellent optical properties. Thus, it can prove to be the best material for manufacture of optoelectronic devices in economic mode. The development of 
newer non-linear optical materials for their possible applications in technological areas like optoelectronics, optical signal processing, optical computing is of crucial importance. Earlier, many efforts have been made to explore the linear and non-linear optical properties of different materials in the bulk state as well as in nanostate. Experimentally, Gupta et al. (2003) have studied the surface optical phonons in GaP nanowires using Raman scattering. Synthesis of $\mathrm{GaP}$ nanowires by heating $\mathrm{Ga}_{2} \mathrm{O}_{3}$ and red phosphor powders has been done by Baoyu et al. (2004). A complete Raman scattering study on GaP nanowires has been done by Chapelle et al. (2005). They gave evidence that Raman spectra are affected by the onedimensional shape of the nanowires. The morphology and microstructure of $\mathrm{GaP}$ nanowires by scanning electron microscopy and transmission electron microscopy have been observed by Xiong et al. (2003). Lee et al. (2003) studied the interband optical transitions in GaP nanowires encapsulated in $\mathrm{GaN}$ nanotubes.

Four different geometrical structures of GaP nanowires are considered and the linear and non-linear optical properties of considered structures are being presented in this paper. The linear and non-linear spectra for considered structures are investigated and finally the optical transition in different energy regions is discussed. The details about the geometrical structures have been given in our earlier published paper (Srivastava et al. 2011). The considered geometrical structures of GaP nanowires are shown in Fig. 1.
Fig. 1 Optimized structures of GaP nanowires. a Two atom linear wire, $\mathbf{b}$ two atom zigzag wire, $\mathbf{c}$ four atom square wire, d six atom hexagonal wire

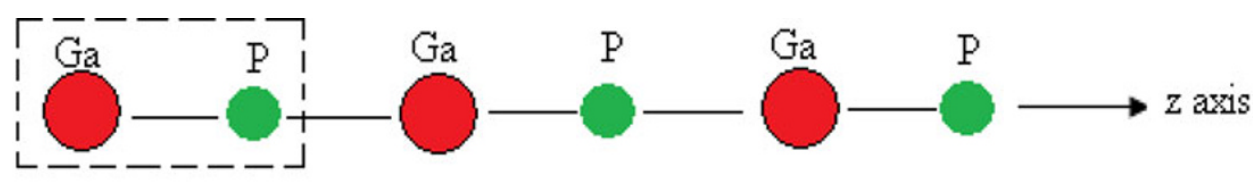

2_atom linear GaP Nanowire

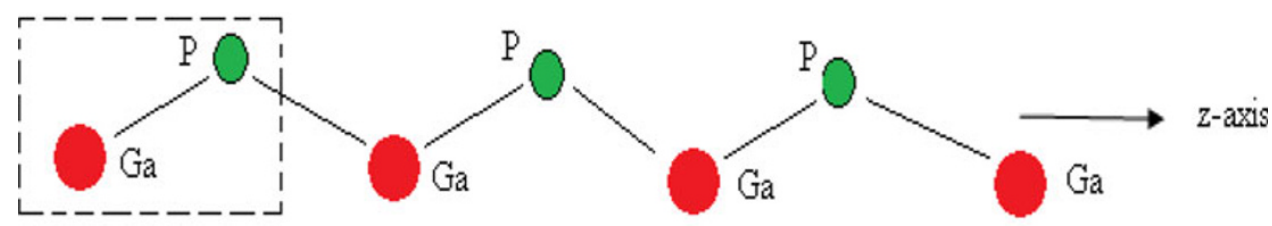

2_atom zigzag GaP Nanowire

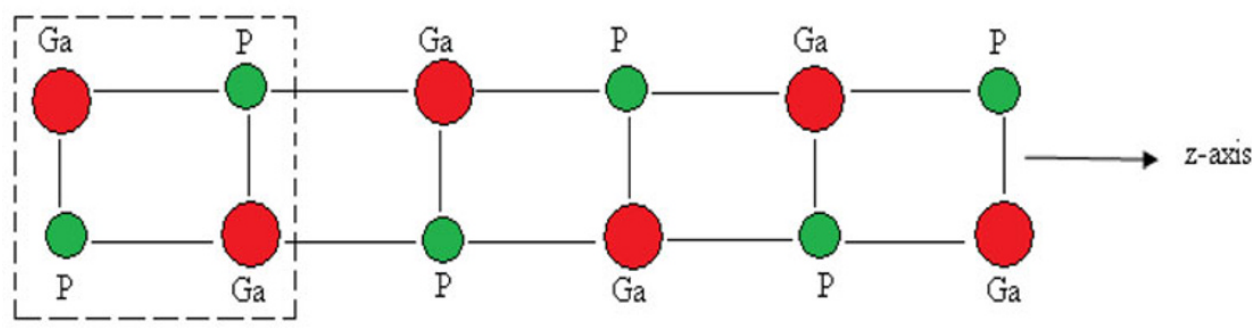

4_atom square GaP Nanowire

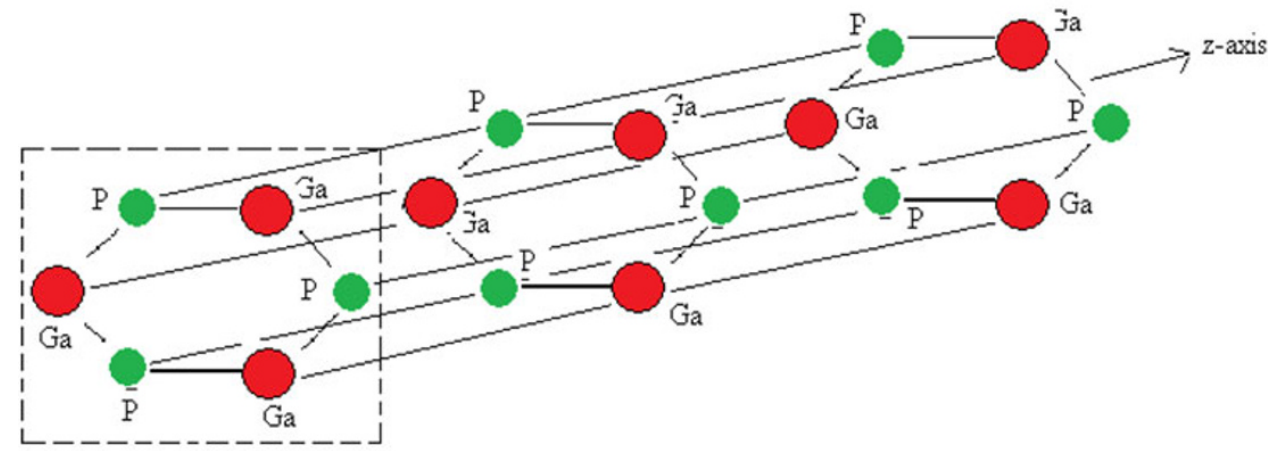

6_atom hexagonal GaP nanowire 


\section{Methodology used}

ABINIT code (Hohonberg and Kohn 1964; Kohn and Sham 1965) has been used for the computational work. The ab initio DFT calculations (Martin 2004; Gonze et al. 2002) are employed within the plane-wave pseudopotential method to investigate the linear and non-linear optical properties of $\mathrm{GaP}$ nanowires. As is evident from the above literature that the pseudopotential method has been very successful in exploring the structural, electronic and optical properties of various materials (Martin 2004); in this calculation, the generalized gradient approximation and the exchange-correlation functional of Perdew, Burke, and Ernzerhof were applied (Perdew et al. 1996). The exchange correlation potential of Troullier and Martins (1991) has been used and these pseudopotentials were taken from ABINIT web page (Gonze et al. 2002). The potentials were tested by performing calculations on bulk $\mathrm{GaP}$ material in which the results were found to be consistent with the experimental ones.

All the calculations were performed in a self-consistent manner. The studied structures were optimized for Hellmann-Feynman forces as small as $10^{-3} \mathrm{eV} / \mathrm{A}$ on each atom and the calculations were performed with a kinetic energy cut off of 30 Hartree. The wires were positioned in a supercell of side 20 au along the $x$ and $y$ directions; the axis of the wire was taken along the $z$ direction and the periodic boundary conditions were applied. The Monkhorst-Pack method with $15 \mathrm{k}$-points sampling along the $z$ direction was used in the integration of the Brillouin zone, all atoms were allowed to relax without any imposed constraint. In order to check the self-consistent calculations, we have determined the self-consistent optimized value for the lattice parameter of bulk GaP materials, the magnitude of atomic relaxation depends on the plane cut-off energy and one has to obtain the convergence with respect to cut-off energy too.

\section{Results and discussion}

The linear and non-linear susceptibility equations have both real and imaginary parts for calculating the optical spectra of any of the nanowires; here, both real and imaginary parts can be important for the gain and loss of energy density in the electromagnetic field at some particular frequency. In the current investigation, only the imaginary part of the susceptibility equation is responsible for the exchange of energy in the electromagnetic field, therefore, the imaginary part of the results so obtained is analyzed.

The imaginary parts of the $z z$ component of the linear dielectric tensor or linear susceptibility for various $\mathrm{GaP}$ nanowires along with bulk are shown in Fig. 2. The linear optical response of bulk seems to be quite smooth, whereas the linear susceptibility gradually increases with energy. In the case of two atom linear wire, response reflects a strong peak around $4.6 \mathrm{eV}$, with some other small and large peaks towards the higher energy side. For two atom zigzag wire structure, a strong absorption with one high magnitude peak at $4.2 \mathrm{eV}$ with several small peaks towards higher energy side is observed. It is found that the four atom square wire linear spectra have the strongest peak around $4.4 \mathrm{eV}$, and there are other weak peaks also at higher energy region. In the case of six atom hexagonal wire structure, the spectra have its highest peak at $4.2 \mathrm{eV}$ but is of less magnitude as compared to that of the other structures; however, there are many other peaks seems in between 4.0 and $6.0 \mathrm{eV}$. In conclusion, the analysis of linear spectra for all considered structures reveals that strongest absorption occurs for the two atom linear wire, two atom zigzag wire and four atom square wire, respectively.

The non-linear response of bulk spectra is as shown in Fig. 3. It can be seen that the total second order susceptibility is zero towards the low energy region up to $4 \mathrm{eV}$. Whereas in the high energy region between the energy levels 4.0 and $6.0 \mathrm{eV}$, the SHG optical spectra are dominated by intra $(2 \omega)$ contribution towards the positive

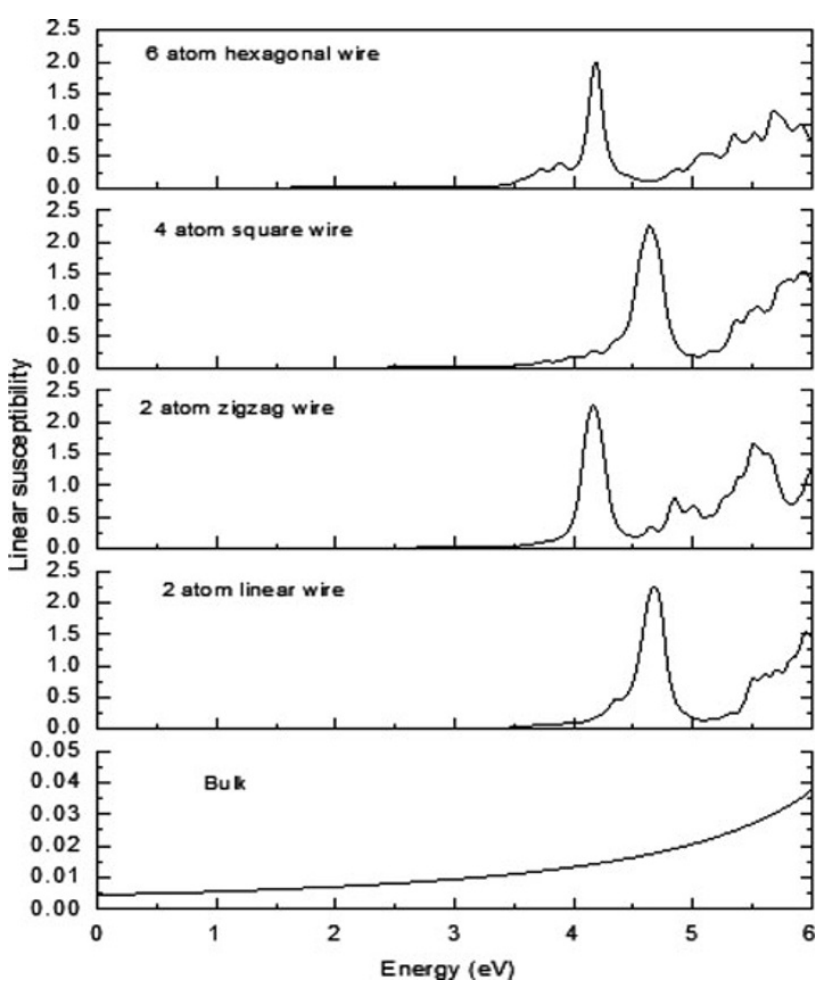

Fig. 2 Calculated imaginary part of the $z z$ component of the linear dielectric tensor for different $\mathrm{GaP}$ nanowires along with bulk $\mathrm{GaP}$ material 


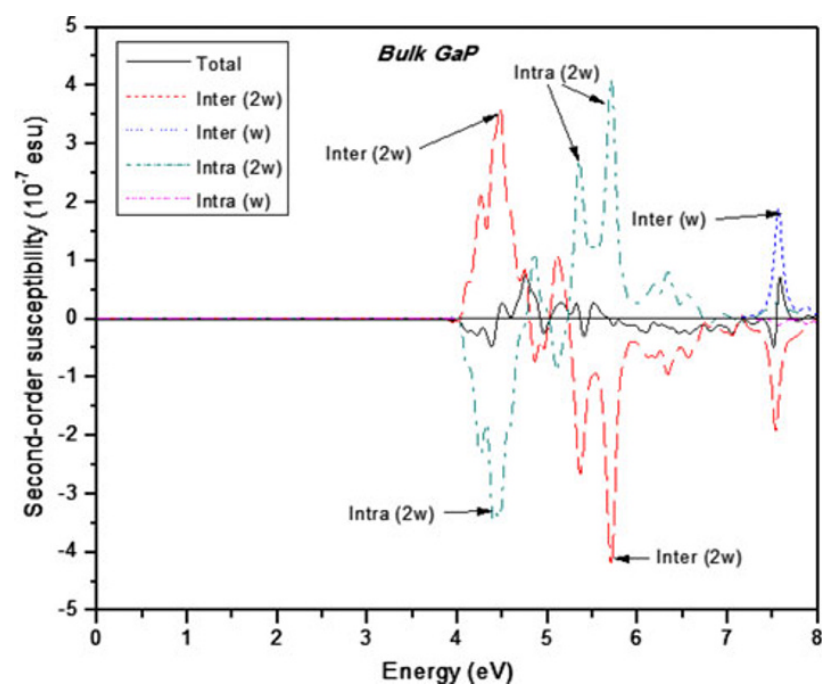

Fig. 3 Calculated second-order susceptibility $\operatorname{Im}\left[\chi_{\mathrm{zzz}}(2 \omega, \omega, \omega)\right]$ and different contribution for bulk GaP material

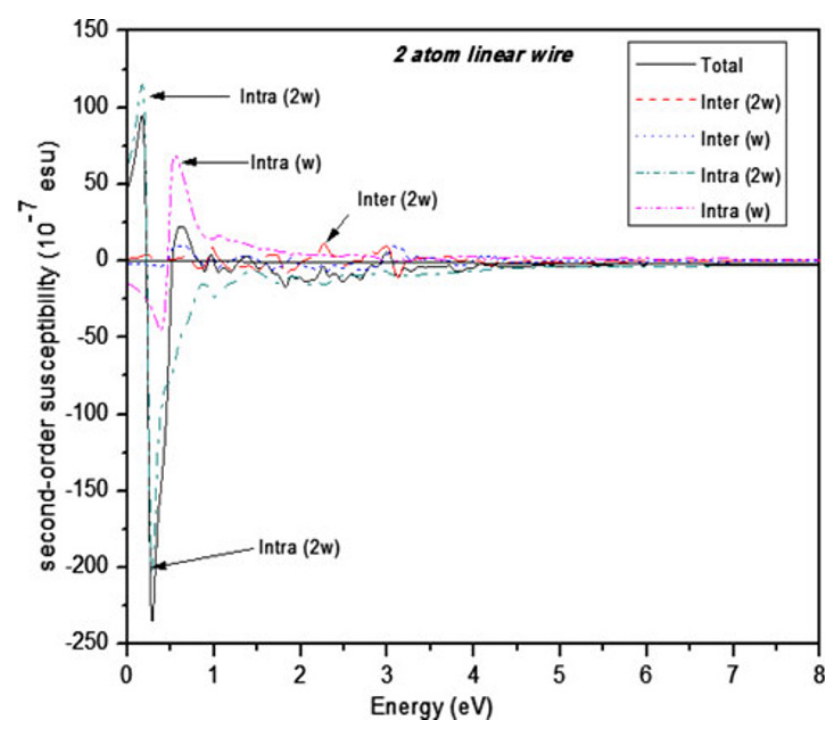

Fig. 4 Calculated second-order susceptibility $\operatorname{Im}\left[\chi_{\mathrm{zzz}}(2 \omega, \omega, \omega)\right]$ and different contribution for two atom $\mathrm{GaP}$ linear wire

susceptibility value and inter $(2 \omega)$ contribution towards the negative susceptibility.

The non-linear optical spectra of different structures of GaP nanowires are presented in Figs. 4, 5, 6 and 7. Various contributions to the imaginary part of $\chi_{\mathrm{zzz}}(2 \omega, \omega, \omega)$ are taken and the non-linear spectra of two atom linear wire are shown in Fig. 4. It is observed that the total SHG peaks are dominant near lower energy values having magnitude of the order of 90 , which reduces almost 7 near $3.2 \mathrm{eV}$ on positive side. On the positive side, major contributions come from intra $(2 \omega)$ transition and intra $(\omega)$ transition, whereas the intra $(2 \omega)$ transition plus the total transition are obtained in the negative region of the graph.

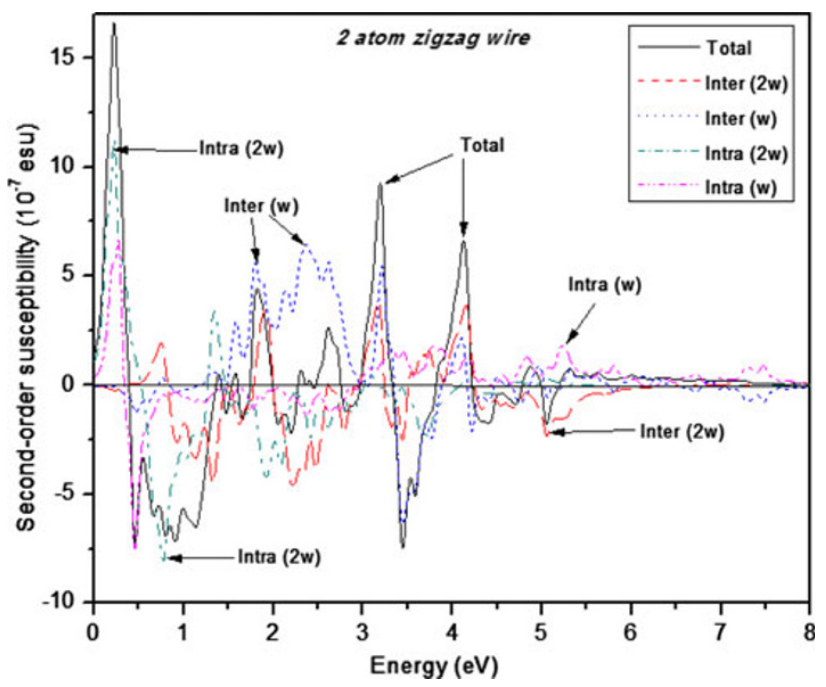

Fig. 5 Calculated second-order susceptibility $\operatorname{Im}\left[\chi_{z z z}(2 \omega, \omega, \omega)\right]$ and different contribution for two atom GaP zigzag wire

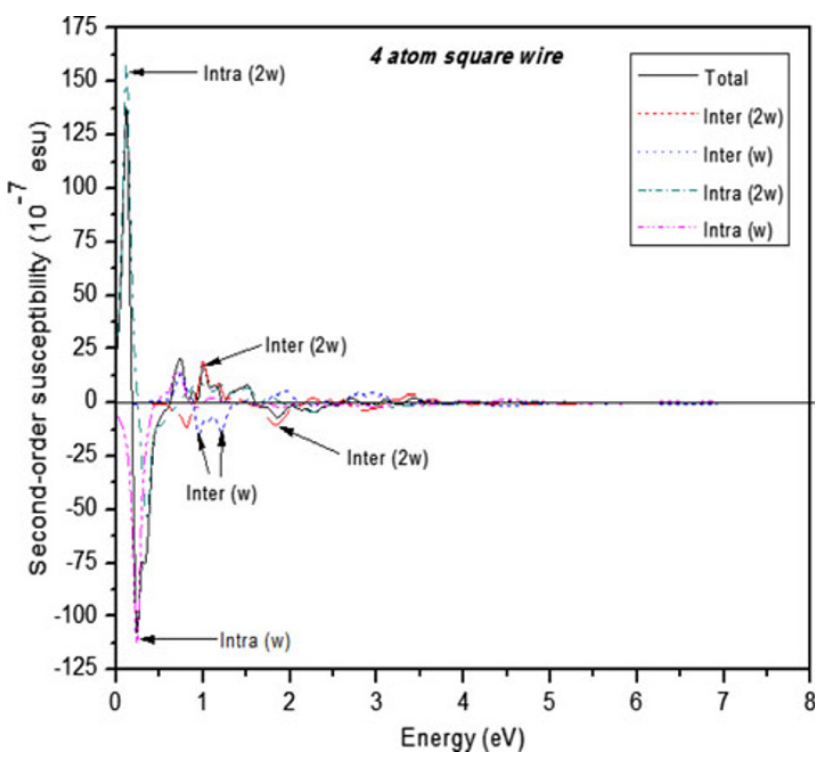

Fig. 6 Calculated second-order susceptibility $\operatorname{Im}\left[\chi_{z z z}(2 \omega, \omega, \omega)\right]$ and different contribution for four atom GaP square wire

The second-order susceptibility values for two atom zigzag wire are seen to be rather complicated as shown in Fig. 5. It can be observed that so many prominent peaks lying between 0.0 and $6.0 \mathrm{eV}$ energy range and it is found that the total SHG susceptibility is dominant near lowest energy region having magnitude of the order of 17 , which reduces almost 2 factors near $5.2 \mathrm{eV}$. Here, the major contributions come from intra $(2 \omega)$ transition, and inter $(\omega)$ transition plus total transition are obtained towards the upper level, whereas the intra $(2 \omega)$ transition and the total transition are obtained towards the lower level.

The non-linear response for a four atom square wire cross section is shown in Fig. 6. The total absorption as 


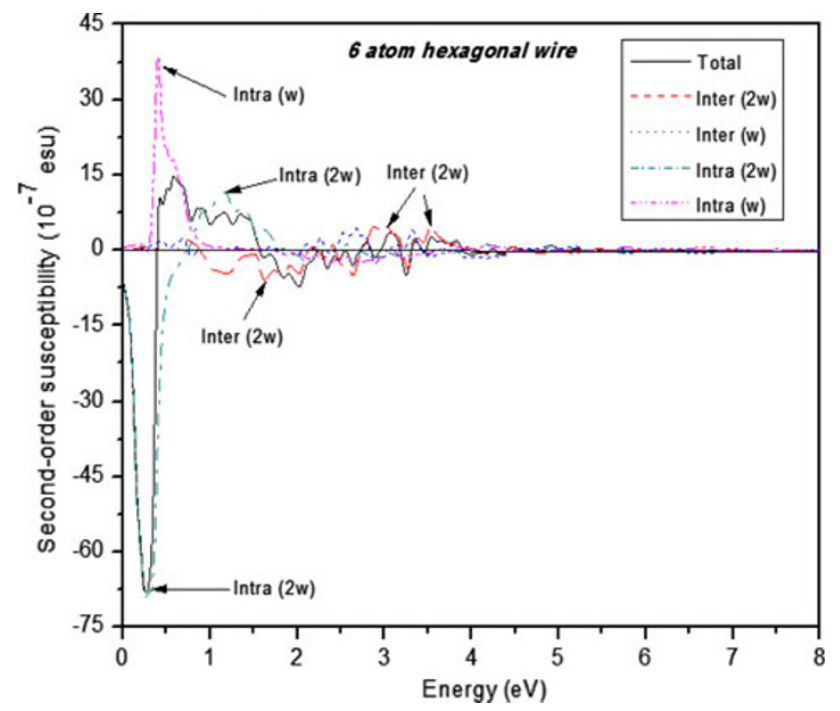

Fig. 7 Calculated second-order susceptibility $\operatorname{Im}\left[\chi_{z z z}(2 \omega, \omega, \omega)\right]$ and different contribution for six atom $\mathrm{GaP}$ hexagonal wire

well as intra $(2 \omega)$ transitions has greater contribution near lower energy region as compared to higher energy region and the magnitude of SHG spectra is high at around 137.5 (total SHG). The highest absorption spectra come from intra $(2 \omega)$ transition and inter $(2 \omega)$ transition in the lower energy region of the positive side, whereas in the negative side SHG spectra, the highest contribution comes from intra $(\omega)$ transition plus total transition.

The optical spectrum for a six atom hexagonal wire is depicted in Fig. 7. It is observed that the total SHG spectra are dominant near lower energy side having the magnitude of the order of 14, which reduces almost 2.5 near $3.7 \mathrm{eV}$ in the positive side. Here, the major contributions come from intra $(\omega)$ transition and inter $(2 \omega)$ transition towards positive side and intra $(2 \omega)$ transition towards negative side having a magnitude of the order of $67.5 \mathrm{eV}$.

Through SHG analysis, it can be concluded that strongest absorption occurs for a four atom square wire configuration. As reported [110] for the same material, it is predicted that four atom square wire structure has the stable structure; thus it can be concluded that stable structure configuration also has the strongest SHG absorption.

\section{Conclusion}

We have calculated and analyzed the linear and non-linear optical response of four different structures of GaP nanowires. The aim of our analysis was to explore the best possible configuration of $\mathrm{GaP}$ nanowire to be applied in photonic and optoelectronic devices. First, we have analyzed the linear response of considered structures. The analysis of linear spectra reveals that strongest absorption occurs for two atom linear wires, two atom zigzag wire and four atom square wires, respectively. Second, we have investigated the SHG susceptibility spectra of these structures. In SHG spectra, we observe remarkable features for all structures. We predict that strongest absorption occurs for four atom square nanowire configuration. We also revealed that the calculated peaks not only get sharper but also show pronounced energy shift. This is mainly due to interband contribution to the imaginary part of the dielectric function. All SHG spectra comprise of total, inter and intra band contributions. The SHG spectra are rather complicated due to various microscopic features observed. We have not come across any of the experimental or theoretical results to compare such type of linear and SHG optical spectra for various structures of $\mathrm{GaP}$ nanowires. The present investigation is important, because size, shape and structure are the important criteria in nano regime, and one must not ignore so far as nanowires are concerned.

Acknowledgments The authors are thankful to Computational Nanoscience and Technology Laboratory (CNTL) of ABV-Indian Institute of Information Technology and Management, Gwalior (M.P.) and Department of Physics, Hindustan College of Science and Technology, Farah, Mathura (U.P.) for providing the infrastructural facilities for computational work.

Open Access This article is distributed under the terms of the Creative Commons Attribution License which permits any use, distribution, and reproduction in any medium, provided the original author(s) and the source are credited.

\section{References}

Baoyu L, Ding W, Qimin D, Jinlei Y (2004) Synthesis and optical study of GaP nanowires. Nanotechnology 15(12):1745-1748

Chapelle MLDLA, Han HX, Tang CC (2005) Raman scattering from GaP nanowires. Eur Phys J B 46:507-513

Gonze X, Beuken JM, Caracas R, Detraux F, Fuchs M, Rignanese GM, Sindic L, Verstraete M, Zerah G, Jollet F, Torrent M, Roy A, Mikami M, Ghosez PH, Raty JY, Allan DC (2002) First-principle computation of material properties: the ABINIT software project. Comput Mat Sci 25:478. http://www.abinit.org

Gupta R, Xiong Q, Mahan GD, Eklund PC (2003) Surface optical phonons in gallium phosphide nanowires. Nano Lett 3(12):1745-1750

Hohonberg P, Kohn W (1964) Inhomogeneous electron gas. Phys Rev 136:B864-B871

Karnik R, Fan R, Yue M, Li D, Yang P, Majumdar A (2005) Electrostatic control of ions and molecules in nanofluidic transistors. Nano Lett 5:943-948

Kohn W, Sham LJ (1965) Self-consistent equations including exchange and correlation effects. Phys Rev 140:A1133-A 1138

Lee MW, Hsueh HC, Lin HM, Chen CC (2003) Interband optical transitions in $\mathrm{GaP}$ nanowires encapsulated in $\mathrm{GaN}$ Nanotubes. Phys Rev B 67:161309(R)

Martin RM (2004) Electronic structure: basic theory and practical methods. Cambridge University Press, Cambridge

Perdew JP, Burke K, Ernzerhof M (1996) Generalized gradient approximation made simple. Phys Rev Lett 77:3865 
Sirbuly DJ, Law M, Yan HQ, Yang PD (2005) Semiconductor nanowires for subwavelength photonics integration. J. Phys. Chem. B 109(32):15190-15213

Srivastava P, Singh S (2008) Linear and second-order optical response of different GaN nanowires. Physica E 40:2742-2746

Srivastava P, Singh S, Mishra A (2011) Electronic Properties of GaP Nanowires of Different shapes. J Nanosci Nanotechnol 11:10464-10469

Troullier N, Martins JL (1991) Efficient pseudopotentials for planewave calculations. Phys Rev B 43:1993-2006
Wang WU, Chen C, Lin KH, Fang Y, Lieber CM (2005) Label-free detection of small-molecule-protein interactions by using nanowire nanosensors. Proc Natl Acad Sci USA 102:3208-3212

Xiong Q, Gupta R, Adu KW, Dickey EC, Lian GD, Tham D, Fischer JE, Eklund PC (2003) Raman spectroscopy and structure of crystalline gallium phosphide nanowires. J Nanosci Nanotechnol 3(4):335-339 